\title{
Biomarkers of venous thromboembolism in cancer: a silent echo from local events?
}

\author{
Emelie Gezelius ${ }^{1} \&$ Mattias Belting ${ }^{* 1,2}$ \\ ${ }^{1}$ Department of Clinical Sciences, Lund University, Skåne University Hospital, Oncology, Barngatan 4, SE-221 85, Lund, Sweden \\ ${ }^{2}$ Department of Immunology, Pathology, \& Genetics, Uppsala University, Rudbecklaboratoriet, SE-751 85, Uppsala, Sweden \\ *Author for correspondence: Tel.: +46 46178 549; mattias.belting@med.lu.se
}

'One obvious reason for the failure to identify risk biomarkers of VTE at the systemic level is the local nature of thrombotic events (except for disseminated intravascular coagulation, a rare event in cancer), and the dynamics and kinetics of the coagulation cascade that may not echo from the future"

First draft submitted: 29 March 2019; Accepted for publication: 2 April 2019; Published online: 29 May 2019

Keywords: anticoagulant treatment $\bullet$ cancer $\bullet$ venous thromboembolism

The relationships between cancer, coagulation activation and venous thromboembolism (VTE) are multifaceted and complex, challenging generations of scientists for over a century. Still, there is no validated biomarker to identify patients at increased risk of VTE, and no convincing evidence that coagulation inhibition improves patient survival.

In 1865 Trousseau described the phenomenon of phlegmasia alba dolens; thrombosis causing a white, painful leg in malignancy [1], and since then the quest has continued to unravel the pathways leading to cancer-associated thrombosis - a major cause of increased morbidity and mortality $[2,3]$. Several of the activated coagulation factors are not only involved in the development of thrombosis, but may also contribute to malignant processes such as angiogenesis and metastasis [4], making them a double-edged sword. Along the same line, anticoagulants have received considerable attention for their tumor-inhibiting effects - both in preclinical and early clinical studies [5].

Although large efforts have been made to find pro-thrombotic biomarkers to predict VTE risk and identify patients that will benefit from prophylactic anticoagulants, no routine screening has been implemented into clinical practice. The purpose of this editorial is to discuss the role of anticoagulants as tumor-inhibiting agents, in the light of recent findings on predictive biomarkers of VTE in cancer.

\section{Anticoagulants as tumor-inhibiting agents}

Apart from preventing and treating VTE, there was evidence to suggest that anticoagulants also have direct tumor-inhibiting properties, for example, by inhibition of metastatic growth and angiogenesis [6]. In 1984, the VA Cooperative study \#75 [7] was the first to show survival benefit with warfarin specifically in small cell lung cancer (SCLC), an aggressive form of lung cancer with 5-year survival rates of less than 10\%. This was followed by two trials that successfully demonstrated improved survival in SCLC with the addition of subcutaneous heparin [8] and dalteparin [9], a low molecular weight heparin (LMWH). The findings from these studies were supported by a meta-analysis concluding that LMWH may increase survival mainly in SCLC [10], indicating that coagulation activation plays an important role in the pathogenesis of this disease.

In conflict with previous studies, more recent findings from three large randomized trials investigating the effects of LMWH in lung cancer populations did not show any evidence of improved survival, despite a reduction of VTE events [11-13]. One possibility is that the negative outcome was due to poor adherence to LMWH treatment. However, in a post hoc analysis of RASTEN, the largest study on a homogenous population of SCLC patients [11], we conclude that the negative results cannot be attributed to inadequate adherence in the LMWH arm 
[Gezelious E ET AL., UNPUBLISHED DATA]. Importantly, the question remains, are there any biomarkers to predict VTE risk and identify a subgroup of patients that still may benefit from LMWH prophylaxis?

In fact, as it now has been established that anticoagulants have limited - if any - tumor-inhibiting effects in the clinical setting and thus should not be used routinely, it is even more important to identify patients at high risk of developing VTE who will benefit from anticoagulants with regard to reduced morbidity.

\section{Biomarkers of VTE}

In a recent substudy of RASTEN [14], we explored various aspects of the coagulation system, particularly focusing on tissue factor (TF) and thrombin, as potential biomarkers of VTE and patient prognosis. TF is the main initiator of the coagulation pathway, forming active complexes with factors VIIa and Xa, further triggering downstream events, including pro-thrombin activation [4]. As a transmembrane glycoprotein, TF can be expressed on cell membranes, but can also be integrated into the lipid bilayer of extracellular vesicles (EV-TF) that is cell-derived, membraneenclosed particles that form an important component of intercellular communication. Cells in stressful conditions, such as hypoxic cancer cells, release EV-TF to a high extent, a large proportion of which are procoagulant in nature [15].

Further down the coagulation cascade, thrombin activity leads to activation and aggregation of platelets as well as fibrin generation, and subsequently to clot formation. Thrombin and TF are also important in signaling events through the activation of PAR, especially PAR1 and PAR2, which trigger signaling pathways involved in metastasis and angiogenesis [4]. Both thrombin and TF, in its various forms, have been widely suggested as biomarkers of cancer-associated thrombosis $[16,17]$.

We did find an association between VTE incidence and total TF, but rather surprisingly there were no correlations to EV-TF or thrombin [14]. This is in contrast to other, albeit very limited, studies claiming promising predictive values of - in particular - EV-TF [17,18]. A strength of our study is the large, homogenous population of SCLC patients from a clinical trial, but one also has to take into consideration the relatively small number of VTE events ( $8 \%$ in the control arm). Furthermore, at odds with expectations from previous studies, increased coagulation activity was not significantly associated with poor outcome in terms of VTE and survival. Instead, we found that patients with low coagulation activity as defined by EV-TF had a worse outcome with the addition of LMWH. Notably, patients with high EV-TF seemed to display improved survival with LMWH, but this could not be statistically confirmed.

\section{Future perspective}

Considering the findings of the RASTEN [11] and the FRAGMATIC [12] studies, showing no survival benefit with additional LMWH despite a significant reduction in VTE, the tumor-inhibiting effects of LMWH in a clinical setting has been called into question. Perhaps more importantly, the results also point out the provocative idea that cancer-associated thrombosis - with a spectrum of manifestations from asymptomatic, incidental findings, to fatal pulmonary emboli - do not contribute to overall mortality to the extent claimed by the current dogma, at least not in lung cancer. Similar results were demonstrated in patients with advanced pancreatic cancer receiving enoxaparin, showing a reduction in VTE events but no difference in survival rates [19].

As a consequence, this should instigate a re-evaluation of the importance of predictive models for VTE in cancer. One obvious reason for the failure to identify risk biomarkers of VTE at the systemic level is the local nature of thrombotic events (except for disseminated intravascular coagulation, a rare event in cancer), and the dynamics and kinetics of the coagulation cascade that may not echo from the future. In our view, future research should focus more on aspects of the coagulation system that are not necessarily related to the final steps of the coagulation cascade and thrombosis. This includes new treatment strategies targeting components of the coagulation system directly related to TF-dependent signaling pathways through, for example, PARs in conjunction with integrins [4]. Another interesting possibility currently being explored in clinical trials is to directly target TF using anti-TF antibody-drug conjugates, especially variants that do not perturb the pro-coagulant function of TF [20]. In this scenario, TF is not targeted in its capacity as the initiator of coagulation, but rather as a tumor surface antigen that can serve as a portal of entry of a cytotoxic drug. Such strategies will require a direct assessment of TF in the target tissue, in other words, in the tumor microenvironment, to allow individualized treatment targeted at TF but not systemic coagulation. 


\section{Financial \& competing interests disclosure}

This work was funded by grants from the Swedish Cancer Fund (CAN 2017/664); the Fru Berta Kamprad Foundations; the Skåne University Hospital donation funds; and the Governmental funding of clinical research within the national health services, ALF. The authors have no other relevant affiliations or financial involvement with any organization or entity with a financial interest in or financial conflict with the subject matter or materials discussed in the manuscript apart from those disclosed.

No writing assistance was utilized in the production of this manuscript.

\section{References}

1. Trousseau A. Phlegmasia alba dolens. Clin. Mèd. de l'Hôtel-Dieu de Paris 3, 654-712 (1865).

2. Blom JW, Osanto S, Rosendaal FR. The risk of a venous thrombotic event in lung cancer patients: higher risk for adenocarcinoma than squamous cell carcinoma. J. Thromb. Haemost. 2(10), 1760-1765 (2004).

3. Sorensen HT, Mellemkjaer L, Olsen JH, Baron JA. Prognosis of cancers associated with venous thromboembolism. N. Engl. J. Med. 343(25), 1846-1850 (2000).

4. Belting M, Ahamed J, Ruf W. Signaling of the tissue factor coagulation pathway in angiogenesis and cancer. Arterioscler. Thromb. Vasc. Biol. 25(8), 1545-1550 (2005).

5. Noble S. Heparins and cancer survival: where do we stand? Thromb. Res. 133(Suppl. 2), S133-S138 (2014).

6. Belting M. Glycosaminoglycans in cancer treatment. Thromb. Res. 133(Suppl. 2), S95-S101 (2014).

7. Zacharski LR, Henderson WG, Rickles FR et al. Effect of warfarin anticoagulation on survival in carcinoma of the lung, colon, head and neck, and prostate. Final report of VA Cooperative Study \#75. Cancer 53(10), 2046-2052 (1984).

8. Lebeau B, Chastang C, Brechot JM et al. Subcutaneous heparin treatment increases survival in small cell lung cancer. "Petites Cellules" Group. Cancer 74(1), 38-45 (1994).

9. Altinbas M, Coskun HS, Er O et al. A randomized clinical trial of combination chemotherapy with and without low-molecular-weight heparin in small cell lung cancer. J. Thromb. Haemostas. 2(8), 1266-1271 (2004).

10. Zhang J, Zhang YL, Ma KX, Qu JM. Efficacy and safety of adjunctive anticoagulation in patients with lung cancer without indication for anticoagulants: a systematic review and meta-analysis. Thorax 68(5), 442-450 (2013).

11. Ek L, Gezelius E, Bergman B et al. Randomized Phase III trial of low-molecular-weight heparin enoxaparin in addition to standard treatment in small-cell lung cancer: the RASTEN trial. Ann. Oncol. 29(2), 398-404 (2018).

12. Macbeth F, Noble S, Evans J et al. Randomized Phase III trial of standard therapy plus low molecular weight heparin in patients with lung cancer: FRAGMATIC trial. J. Clin. Oncol. 34(5), 488-494 (2016).

13. Meyer G, Besse B, Doubre $\mathrm{H}$ et al. Anti-tumour effect of low molecular weight heparin in localised lung cancer: a Phase III clinical trial. Eur. Respir. J. 52(4), 1-10 (2018).

14. Gezelius E, Flou Kristensen A, Bendahl PO et al. Coagulation biomarkers and prediction of venous thromboembolism and survival in small cell lung cancer: a sub-study of RASTEN - a randomized trial with low molecular weight heparin. PloS ONE 13(11), e0207387 (2018).

15. Svensson KJ, Kucharzewska P, Christianson HC et al. Hypoxia triggers a proangiogenic pathway involving cancer cell microvesicles and PAR-2-mediated heparin-binding EGF signaling in endothelial cells. Proc. Natl Acad. Sci. USA 108(32), 13147-13152 (2011).

16. Ay C, Dunkler D, Simanek R et al. Prediction of venous thromboembolism in patients with cancer by measuring thrombin generation: results from the Vienna Cancer and Thrombosis Study. J. Clin. Oncol. 29(15), 2099-2103 (2011).

17. Khorana AA, Francis CW, Menzies KE et al. Plasma tissue factor may be predictive of venous thromboembolism in pancreatic cancer. $J$. Thromb. Haemos. 6(11), 1983-1985 (2008).

18. Manly DA, Wang J, Glover SL et al. Increased microparticle tissue factor activity in cancer patients with venous thromboembolism. Thromb. Res. 125(6), 511-512 (2010).

19. Pelzer U, Opitz B, Deutschinoff G et al. Efficacy of prophylactic low-molecular weight heparin for ambulatory patients with advanced pancreatic cancer: outcomes from the CONKO-004 trial. J. Clin. Oncol. 33(18), 2028-2034 (2015).

20. Theunissen JW, Cai AG, Bhatti MM et al. Treating tissue factor-positive cancers with antibody-drug conjugates that do not affect blood clotting. Mol. Cancer Ther. 17(11), 2412-2426 (2018). 
